\title{
Prognostic Value of Hedgehog Signal Component Expressions in Hepatoblastoma Patients
}

\author{
Ying-Cun Li1 ${ }^{1}$, Yu-Hua Deng' ${ }^{1}$, Zhen-Hua Guo ${ }^{1}$, Ming-Man Zhang ${ }^{1}$, Jin Zhu' ${ }^{2}$, Chon-Lun Pu${ }^{1}$, \\ Chun-Ping Xiang ${ }^{2}$, Chun-Bao Guo ${ }^{1}$ \\ ${ }^{1}$ Department of Hepatobiliary Surgery, Children's hospital, Chongqing Medical University, Chongqing, P.R. China, \\ ${ }^{2}$ Department of pathology, Children's hospital, Chongqing Medical University, Chongqing, P.R. China
}

\begin{abstract}
Objective: Activation of hedgehog (Hh) pathway has been implicated in the development of human malignancies. Hh as well as related downstream target genes has been extensively studied in many kinds of malignant tumours for clinical diagnostic or prognostic utilities. This study aimed at investigating whether $\mathrm{Hh}$ molecules provides a molecular marker of hepatoblastoma malignancy.

Methods: We obtained tissue sections from 32 patients with hepatoblastoma as well as cholestasis and normal control. Immunohistochemical analysis were performed to determine Hh signal components in human hepatoblastoma. The prognostic significance of single expression of $\mathrm{Hh}$ signal components were evaluated using Cox proportional hazards regression models and Kaplan-Meier survival analysis for statistical analysis.

Results: Expression of Hh signal components showed an increase in hepatoblastoma compared with cholestasis and normal tissues. There was a positive correlation between Smo or Gli1 expression and tumor clinicopathological features, such as histological type, tumor grade, tumor size and clinical stage. Both Smo or Gli1 protein high expression was significantly associated with poor prognosis by univariate analyses and multivariate analyses.

Conclusions: Abnormal Hh signaling activation plays important roles in the malignant potential of hepatoblastoma. Gli1 expression is an independent prognostic marker.
\end{abstract}

Kew words: Hedgehog (Hh); Hepatoblastoma; Prognosis; Indicator

Abbreviations: Hh: hedgehog; Shh: Sonic hedgehog; Smo, Smoothened; Gli-1: glioma-associated oncogene homologue 1; Ptch1: patched1.

\section{INTRODUCTION}

Hepatoblastoma is one of the commonly diagnosed pediatric tumours all over the world. The prognosis of the patients with hepatoblastoma is various. Presently, there are few clues as to its pathogenesis and biological characteristics [1, 2, 3]. Except for stage of disease, there are few markers to predict the prognosis of pa-

Ying-Cun Li and Yu-Hua Deng contributed equally to this paper. tients or to evaluate the malignant grade of hepatoblastoma. Elucidation of the useful prognosis-predicting factors is necessary to improve the prognosis of patients with hepatoblastoma. One area of intense research is the identification of molecular markers to fully assess the prognosis of patients and to define innovative strategies. It has revealed that activation and inactivation of specific molecular markers plays critical roles in hepatoblastoma and other malignant diseases $[4,5]$. This plays an important role in the pathogenesis of hepatoblastoma, but is not considered to be a good molecular marker to distinguish high-risk tumours from others [6, 7]. Expanded use of these markers for the diagnosis and individualization of therapy, however, has been hampered by a lack of universal acceptance of their prognostic significance. There is still no consensus in clinical practice on how to evaluate the effectiveness of candidate molecular markers in evaluating its prognostics.

Recently, a series of studies reported that the $\mathrm{Hh}$ signaling pathway is involved in differentiation and progression of a variety of malignant processes, including both solid tumors and hematopoietic neoplasms $[8,9,10]$. Hedgehog $(\mathrm{Hh})$ pathway is a system that is known to regulate the viability and differentiation of various types of progenitors. Hh ligands are soluble, lipid-modified morphogens that interact with patched (Ptc), a membrane-spanning receptor on the surface of $\mathrm{Hh}$ responsive cells. This ligand-receptor interaction prevents Ptc from inhibiting its coreceptor, smoothened (Smo), a member of the seven transmembrane-receptor family. Activated Smo, in turn, initiates a series of intracellular events that culminate in activation and nuclear localization of glioblastoma (Gli) family transcription factors, which is associated with development of basal cell carcinomas and medulloblastomas. Signaling is turned off when the level of Ptc exceeds that of Hh ligands, permitting "free" Ptc to interact with and inhibit Smo [11].

Although few reports have shown blocking the hedgehog pathway inhibits hepatoblastoma growth. There is no definitive data to accurately predict a correlation between Hh pathway and the clinicopathological features of hepatoblastoma and long-term outcome in patients. To evaluate the role of hedgehog pathway as factor to provide a molecular marker of hepatoblastoma neoplastic process and biologic behavior of the tumor. The present study, for the first time to our knowledge, combined molecular analysis of Hh pathway in 
hepatoblastoma specimens and clinicopathological features as well as outcomes of the patients in a molecular epidemiological study of a Chinese population.

\section{MATERIALS AND METHODS}

\section{Patients Characteristics and Tissue Samples}

Hepatoblastoma tissue samples were obtained from 32 patients who were referred to the affiliated hospital of Chongqing Medical University, Chongqing, P. R. China, with detailed clinical data. All subjects were ethnic Chinese. The patients with these tumours were treated under the framework of the guideline from Chinese Medical Association. The clinical stages of disease were determined at the time of initial biopsy or resection according to the classification of the Chinese Medical Association of Pediatric Surgeons. Histological subtypes were diagnosed according to the classification of Haas et al. [12]. Control liver tissues was obtained from cholestatic disease patients with comparable age, including patients who had severe cholestasis, diagnosed with choledochal cysts (CDCs) at the time of cyst excision $(n=4)$. Cholestatic disease were chosen because of similiarities of Hh pathway activition reported. Normal controls included 5 percutaneous liver biopsy under general anesthetic to rule out Glycogen storage disease. All diagnoses were based on clinical and laboratory data and on histopathological examination of histological samples. This study was performed according to a protocol approved by the Institutional Review Board of the Chongqing Medical University, Chongqing, Peoples Republic of China in accordance with the ethical standards prescribed by the Helsinki Declaration of the World Medical Association. Written informed consent was obtained from the parents prior to specimen collection. The patients were followed every 3 months by clinical examination and basic laboratory tests during the first three years from diagnosis and then every 6 months. The median follow-up in our study is 55 months (range 39-60 months). Survival was calculated at the time from follow-up to death from any cause for overall survival. For overall survival, all deaths, irrespective of cause, were considered events. Data concerning the patients who died from other causes or who were still alive at the end of our study were censored.

\section{IMMUNOHISTOCHEMISTRY}

Contiguous 4- $\mu \mathrm{m}$ formalin-fixed, paraffin-embedded sections were firstly submitted to rehydrate by incubating successively in 100\%, 90\%, and 70\% ethanol. After deparaffinizating to water, heat-induced epitope retrieval was unmasked in citrate buffer with steam for $60 \mathrm{~min}$. Sections were then incubated overnight at 4 with an optimal dilution $(0.1 \mathrm{mg} / \mathrm{L})$ of a primary polyclonal goat antibody against human Shh (N-19), Ptc (C-20), Smo (N-19) and Gli-1 (C-18) (1:160 dilution, mouse clone, Santa Cruz Biotechnology Inc., Santa Cruz, CA). Next, the tissue sections were incubated with a 1:300 dilution of biotinylated secondary antibody for both proteins goat antimouse immunoglobulins (Shanghai Sangon Biotech, Shanghai, China). After development of the color with diaminobenzidine, the slides were counterstained with hematoxylin. Negative control slides were run with every batch, which included an isotype-matched immunoglobulin at the same concentration as that of the primary antibody. Basal cell carcinoma tissue served as the internal positive control for Shh, Ptc, Smo and Gli-1 immunoreactivity. Under standard light microscopy at_400, single neoplastic cell positive cytoplasmic and nuclear staining was defined as described previously [13]. Cytoplasmic- and nuclear-positive cells of average 20 fields per tumor(100 cells per field) from typical section were counted and submitted to positive rates. All Immunohistochemical slides were performed independently by two investigators (Jin Zhu and Chunping Xiang). If positive rate exceed $5 \%$, immunostaining result were consided positive for this patient.

\section{STATISTICAL ANALYSIS}

Statistical comparisons were carried out using SPSS software for Windows (SPSS, Inc., Chicago, IL). Pearson's $\chi^{2}$ test or Fisher's exact test was used to examine correlations between the Hh signal staining percentages and the clinicopathologic features. The overall survival curves for each group of patients were estimated by the Kaplan-Meier method and the resulting curves were compared using the Cox-Mantel test. Univariate and multivariate Cox proportional hazards regression models were used to examine prognostic value of the Smo or Gli1 staining, and other clinicopathologic features. Survival curves were constructed using the KaplanMeier method and compared using the log-rank test. The level of significance was set at $p<0.05$.

\section{RESULTS}

\section{Hh Pathway Components Expression in Hepatoblastoma Tissues}

To investigate if $\mathrm{Hh}$ pathway were changed with human hepatoblastoma development and metastasis, Hh pathway components expression analyses were carried out by immunohistochemistry. Fig. 1 shows the representative profile of immunostaining for Shh, Patched, Smo and Gli1 in both the cytoplasm and nuclei. Shh expression was heterogenous and localized to the cytoplasm, stroma and glandular lumen of tumor. Ptc and Smo were localized to the cytoplasm of neoplastic epithelium and cell membrane, and a heterogeneous expression pattern of Gli1 in the cancer group was observed. Gli-1 was mainly localized to the cytoplasm and scattered nucleus of hepatoblastoma tumor cells. Shh protein expression was present in almost all tumors at different levels and had highest expression in cancer groups. The results from immunohistochemical analysis of $\mathrm{Hh}$ pathway components with regard to the malignant potential of hepatoblastoma are summarized in Table 1. Elevated expression of Shh was observed in $87.5 \%(28 / 32)$ of hepatoblastoma, whereas its expression was weak or undetectable in cholestasis $(\mathrm{P}=0.040$, Chi-square $)$ and normal tissues $(\mathrm{P}=$ 0.008 , Pearson's $\chi^{2}$ test). Patched and Smo expression were also intensively expressed in hepatoblastoma, they were observed very weakly in any cholestasis and normal tissues. no significant difference in their pro- 

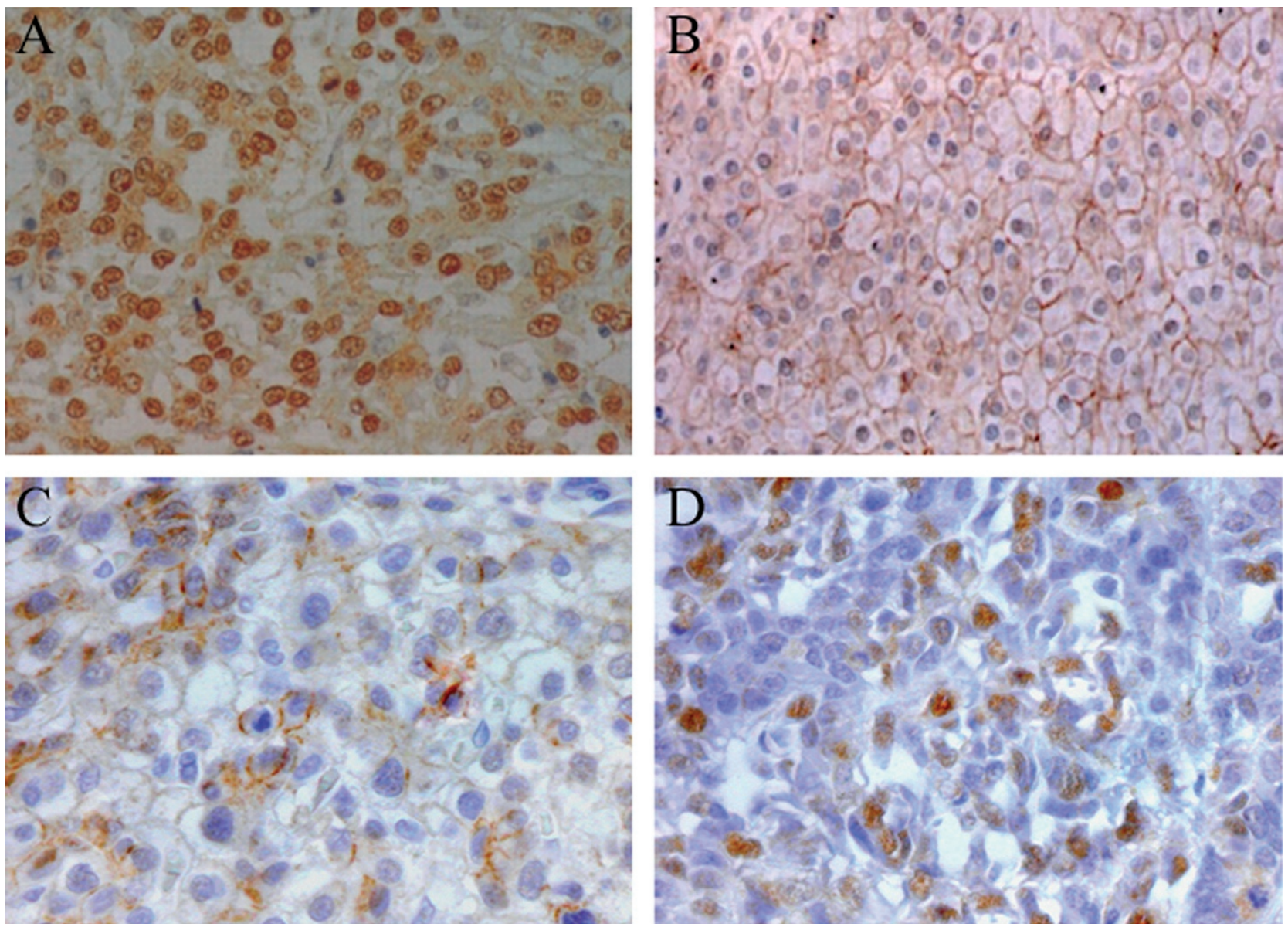

Fig. 1. A representative series of immunohistochemical staining of Hh signaling components from hepatoblastoma patients. (A) Immunohistochemistry for CK-7 expression (expected deeply-brown) in liver sections from a child with BA demonstrating the expression of Shh was present in the cytoplasm of epithelium and stroma tissues. (B) Patched and (C) Smo expression was observed in cytoplasm and cell membrane of tumors. (D) Gli1 expression was scattered found in nuclear of hepatoblastoma cells.

tein expressions was found between hepatoblastoma and cholestasis tissues $\left(\mathrm{P}=0.143\right.$, Pearson's $\chi^{2}$ test). The expression of Gli1 protein in the cytoplasm also increased in tumor tissues compared with that in the cholestasis and normal liver tissues (Table 1, Pearson's

Table 1. Hh pathway components staining status in hepatoblastoma, cholestasis tissues and control.

\begin{tabular}{|c|c|c|c|c|c|}
\hline \multirow[t]{2}{*}{ Variables } & \multirow{2}{*}{$\begin{array}{l}\text { hepato- } \\
\text { blastoma } \\
(n=32)\end{array}$} & \multirow{2}{*}{$\begin{array}{l}\text { cholestasis } \\
\text { tissues } \\
(n=12)\end{array}$} & \multicolumn{3}{|c|}{ Control } \\
\hline & & & $P$ & $(n=8)$ & $P$ \\
\hline \multicolumn{6}{|l|}{ Shh } \\
\hline$(+)$ & 28 & 7 & 0.040 & 3 & 0.008 \\
\hline$(-)$ & 4 & 5 & & 5 & \\
\hline \multicolumn{6}{|l|}{ Gli-1 } \\
\hline$(+)$ & 22 & 6 & 0.143 & 2 & 0.032 \\
\hline$(-)$ & 10 & 6 & & 6 & \\
\hline \multicolumn{6}{|l|}{ Smo } \\
\hline$(+)$ & 21 & 7 & 0.245 & 2 & 0.041 \\
\hline$(-)$ & 11 & 5 & & 6 & \\
\hline \multicolumn{6}{|l|}{ Ptc } \\
\hline$(+)$ & 26 & 8 & 0.181 & 4 & 0.075 \\
\hline$(-)$ & 6 & 4 & & 4 & \\
\hline
\end{tabular}

$\chi^{2}$ test). There was significant correlation between Gli1 and Patched or Smo expression. There was also a positive correlation between Patched expression and Smo expression in hepatoblastoma (Data not shown).

\section{Clinicopathologic Features of Gli-1 Staining}

The clinicopathologic features of the 32 hepatoblastoma cases with theirs Hh pathway components staining data are summarized in Table 2. Of the 32 hepatoblastoma patients, Gli-1 overexpression correlated with different histological type $(\mathrm{P}=0.046)$, tumor size $(\mathrm{P}=0.030)$ and clinical stage $(\mathrm{P}=0.000)$ respectively, whereas Smo overexpression correlated with clinical stage $(\mathrm{P}=0.010)$, Preoperative chemotherapy $(\mathrm{P}=$ $0.022)$ and prognosis $(\mathrm{P}=0.022)$. The levels of Smo and Gli-1 in tumours obtained after preoperative chemotherapy did significantly differ from those in tumours obtained without any therapies. The levels of Smo and Gli-1 significantly increased in advanced stages (stages IIIA, IIIB, and IV, P=0.010 and 0.000, respectively). There was no significant correlation between the levels of serum AFP and Gli-1 (Data not shown). There were no significant associations of Gli1 staining with gender, age $(\mathrm{P}>0.05$ for all of the analyses). 
Table 2. Association between Shh, Gli1 and Gli-1 staining and pathological characteristics of hepatoblastoma patients (Pearson's $\chi^{2}$ test).

\begin{tabular}{|c|c|c|c|c|c|c|}
\hline Prognostic factors & $\begin{array}{l}\operatorname{Shh}(+) \\
\mathrm{n}(\%)\end{array}$ & $P$ & $\begin{array}{c}\operatorname{Smo}(+) \\
\mathrm{n}(\%)\end{array}$ & $P$ & $\begin{array}{l}\text { Gli-1(+) } \\
\text { n( }(\%)\end{array}$ & $P$ \\
\hline $\begin{array}{l}\text { Age }(\mathrm{y})(\mathrm{n}) \\
0-2 \text { years }(15) \\
2-5 \text { years }(12) \\
\text { Over } 5 \text { years }(5)\end{array}$ & $\begin{array}{r}13 \\
11 \\
4\end{array}$ & 0.554 & $\begin{array}{r}10 \\
8 \\
3\end{array}$ & 0.508 & $\begin{array}{r}10 \\
9 \\
3\end{array}$ & 0.571 \\
\hline $\begin{array}{l}\text { Gender } \\
\text { Female (10) } \\
\text { Male (21) }\end{array}$ & $\begin{array}{r}9 \\
19\end{array}$ & 0.704 & $\begin{array}{r}5 \\
16\end{array}$ & 0.148 & $\begin{array}{r}7 \\
15\end{array}$ & 0.625 \\
\hline $\begin{array}{l}\text { Histology } \\
\text { Well (17) } \\
\text { Poorly (15) }\end{array}$ & $\begin{array}{l}13 \\
15\end{array}$ & 0.07 & $\begin{array}{r}9 \\
12\end{array}$ & 0.108 & $\begin{array}{r}9 \\
13\end{array}$ & 0.046 \\
\hline $\begin{array}{l}\text { Tumor size } \\
\qquad \begin{array}{l}\mathrm{n} \leq 2(3) \\
2<\mathrm{n} \leq 5 \\
5<\mathrm{n}(15)\end{array}\end{array}$ & $\begin{array}{r}2 \\
11 \\
15\end{array}$ & 0.056 & $\begin{array}{r}1 \\
8 \\
12\end{array}$ & 0.070 & $\begin{array}{r}1 \\
8 \\
13\end{array}$ & 0.030 \\
\hline $\begin{array}{l}\text { Stage } \\
\text { Stages I, II (8) } \\
\text { StagesIII, IV (24) }\end{array}$ & $\begin{array}{r}5 \\
23\end{array}$ & 0.078 & $\begin{array}{r}2 \\
19\end{array}$ & 0.010 & $\begin{array}{r}1 \\
21\end{array}$ & 0.000 \\
\hline $\begin{array}{l}\text { Preoperative chemotherapy } \\
\text { Yes(17) } \\
\text { No }(15)\end{array}$ & $\begin{array}{l}14 \\
14\end{array}$ & 0.350 & $\begin{array}{r}8 \\
13\end{array}$ & 0.022 & $\begin{array}{r}9 \\
13\end{array}$ & 0.046 \\
\hline $\begin{array}{l}\text { Prognosis } \\
\quad \text { Survived with evidence-free (19) } \\
\text { Recurrence/died of disease (13) }\end{array}$ & $\begin{array}{l}15 \\
13\end{array}$ & 0.108 & $\begin{array}{r}8 \\
13\end{array}$ & 0.022 & $\begin{array}{l}10 \\
12\end{array}$ & 0.020 \\
\hline
\end{tabular}

\section{Prognostic Value of Smo And Gli1}

To evaluate the prognostic value of Smo and Gli1, we then carried out Cox regression analyses, we performed a log-rank test followed with Kaplan-Meier estimates. The overall Kaplan-Meier survival curves for Smo and Gli1 staining expression are shown in Fig. 2. The overall Kaplan-Meier survival curves of all patients (Fig. 2A) show that the 5-year survival rate in the patients with Gli1 pisitive expression was 53\%, while that in the remaining patients was approximately $72 \%$. The prognosis of the patients with positive expression of Gli1 was significantly worse than that of other patients. Similarly, patients with positive expression of Smo $(n=21)$ also had poorer survival than those with negative Smo expression $(n=11)$. Since the levels of Gli1 were significantly correlated with advanced stages of tumour, the correlation between Gli1 expression and prognosis was examined in tumours in early stages (stage I or II), and those in advanced stages (stage III or IV), separately. The prognosis of the 21 advanced stages patients with high levels of Gli1 expression was significantly poor in advanced tumours. Among them 12 patients with Gli-1 nuclear $( \pm)$ expressing cancers died from distant metastasis within a few months. Three (16.7\%) of the 24 advanced cases with low Gli-1 expression remain disease-free. In 8 patients with early tumours, all 7 patients with low levels of Gli1 expression are alive disease free and one out of eight patients with high levels of Gli1 expression showed poor prognosis. One of the 8 cases with early stage hepatoblastoma showed
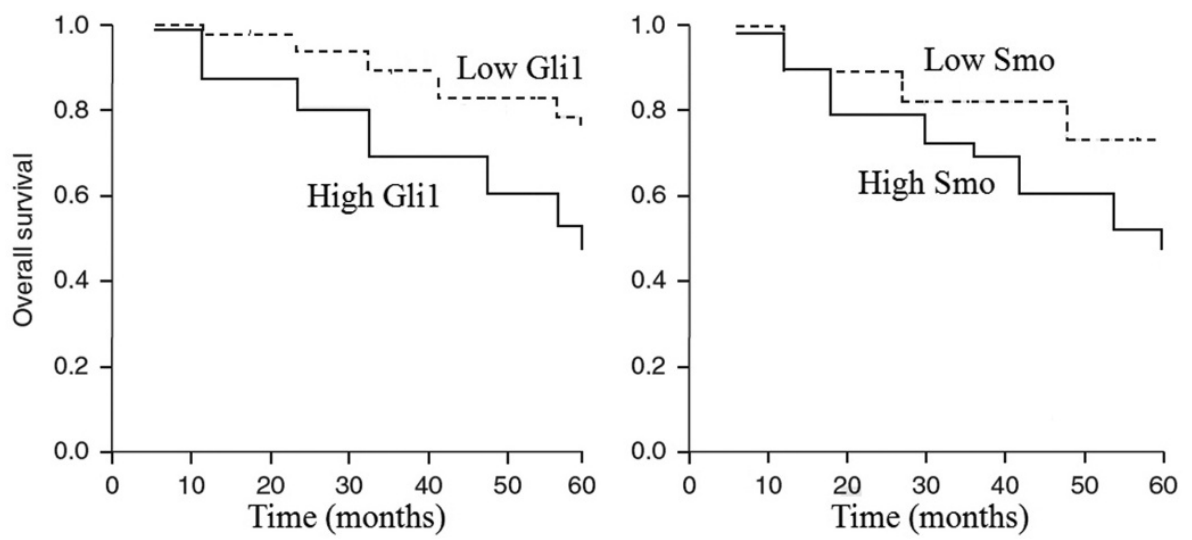

Fig. 2. Kaplan-Meier curves for overall survival of the patient population with hepatoblastoma. A, Overall survival according to Gli1 status. patients with nuclear Gli1 (+) showed poorer survival rates than those with tumors expressing Gli1 (-) $(\mathrm{P}<0.01, \log -$ rank test). B, Survival according to the levels of Smo expression. Patients with Smo (+) pattern showed poorer outcome than the remainder of the population $(\mathrm{P}<0.01)$. 
Table 3. Univariate and Multivariate analysis of potential molecular markers for overall survival of hepatoblastoma.

\begin{tabular}{lllll}
\hline & Univariate & $\mathrm{P}$ & Multivariate HR $(95 \% \mathrm{CI}) *$ & $\mathrm{P}$ \\
\hline $\begin{array}{c}\text { Preoperative chemotherapy } \\
\text { (yes versus no) }\end{array}$ & $1.36(0.89-3.79)$ & 0.046 & $2.16(0.91-5.11)$ & 0.074 \\
$\begin{array}{c}\text { surgical resection } \\
\quad \text { yes versus no) }\end{array}$ & $1.81(0.92-3.62)$ & 0.082 & $2.13(1.17-4.43)$ & 0.033 \\
$\begin{array}{c}\text { Gli1 expression } \\
\text { (positive versus negative) }\end{array}$ & $4.75(1.16-15.61)$ & 0.011 & $6.34(1.35-18.86)$ & 0.007 \\
$\begin{array}{c}\text { Patched expression } \\
\text { (positive versus negative) }\end{array}$ & $2.53(0.81-5.86)$ & 0.161 & $3.12(0.93-6.45)$ & 0.084 \\
\hline
\end{tabular}

* Adjusted for tumor grade, histologic type, and stage classification

recurrence of tumours, and showed high expression of Shh. These results indicate that a significant inverse relationship of Gli1 expression with a poor prognosis was observed during a 5-year follow-up and suggest that Gli1 expression is a valuable biomarker of prognosis.

\section{Multivariate Analysis of Gli1 Expression}

To identify which independent factors had a significant influence on survival, multivariate analysis of overall survival using the Cox proportional hazards model was further performed to determine the prognostic value of Smo and Gli1 expression when adjusted with other prognostic factors. For this multivariate analysis, variables with P-value lower than 0.30 in the univariate analysis were also selected: tumor grade, histologic type, curative surgery, preoperative chemotherapy and stage classification. As stage classification was significantly associated with distant metastasis, we used stage classification in this multivariate analysis. Cox's regression analysis showed that Gli1 was an independent prognostic factor adjusted by tumor grade, stage, histologic type and patients' age (Table 3, $P<0.01)$. Survival curves were also confirmed this results. For preoperative chemotherapy, the difference in tumor survival lost statistical significance in the multivariate analysis.

\section{DISCUSSION}

Uptonow, there is no report concerning prognosis implications of Hh signaling pathway with hepatoblastoma. Our data revealed that Hh pathway components are active in hepatoblastoma patients, which is in accordance with previous data about a variety of human cancers $[14,15]$. In the current study, besides confirming the increased expression of Shh, Patched, Smo and Gli1 proteins in hepatoblastoma compared with cholestasis liver tissue, we also found for the first time, that hepatoblastoma patients who were high expression for $\mathrm{Hh}$ pathway components showed poorer prognosis than those who were low expression for $\mathrm{Hh}$ pathway components. This appears to be the first attempt to link Hh pathway components with outcome of hepatoblastoma patients, in particular, Gli1 was independent of other clinicopathological parameters.

Hh signaling pathway controls many aspects of cell differentiation, tissue patterning and embryonic development [16]. In normal tissues, Hh-induced progeni- tor-cell proliferation is transient and tightly regulated, resulting in acute epithelial repair and regeneration after injury. In contrast, aberrant activation of tumour Hh signalling cascades is not controlled by regulatory mechanisms, leading to support survival of tumour clonogens and tumour regrowth in an autocrineparacrine manner $[17,18]$. Aberrant activation of the pathway was reported in a variety of human cancers. It have already been reported a significant correlation between high $\mathrm{Hh}$ pathway activity and poor outcomes of patients and such tumours showed a high recurrence rate. In childhood tumours, Hh pathway were detected in a majority of cases of intestinal stromal tumors [19]. However, the role of the Hh pathway in human hepatoblastoma is still unclear. In the current study, we investigated the expression of $\mathrm{Hh}$ pathway components including Shh, Ptc, Smo and Gli-1 using immunohistochemical techniques. Our data demonstrated that Shh and Gli-1 were expressed at higher levels in hepatoblastoma than in cholestasis and normal liver tissue. The expression of Shh correlated with high risk hepatoblastoma and large tumor sizes.

The signals that can influence migratory potential are thought to result in highly aggressive cancer forms and thereby contribute to cancer recurrence Since all stage 4 cases underwent different chemotherapeutic regimen, one explanation for this result is that chemotherapy might have not been effective in preventing recurrence in these four early cases. Indeed, postoperative adjuvant chemotherapy failed to improve the prognosis of patients with Gli-1 nuclearpositive tumours. Any therapeutic intervention could not arrest the disease progression in association with Hh signal activation. Thus, in early stage tumours, selection of patients for chemotherapy based on high Hh activity might be an effective method to improve the prognosis of this category of patient. Moreover, the exclusion of low-risk patients from postoperative chemotherapy could spare some of its serious side effects. These results suggest that Hh signal activation might promote cancer regrowth and progression after chemotherapy, and that subtle Gli-1 activation is indicative of the emergence of 'more aggressive' cancer cells. Hh activation even after chemotherapy correlated with earlier relapse and treatment resistance. The useful biological prognostic factors are of great clinical importance for discriminate a subgroup of hepatoblastoma with more aggressive biological properties, which correlate with a poor prognosis. Hh pathway ac- 
tivity may correlate with malignant potential in childhood malignant tumour hepatoblastoma.

To identify high-risk patients with hepatoblastoma, we also documented a heterogeneous protein expression pattern of Hh signal components in the cancer group. Our results also suggest that Patched and Gli1 protein overexpression were correlated with poor prognosis of patients, which were also suggested in other cancers [19, 20]. Gli-1 nuclear expression might therefore be a useful and reliable biomarker for the screening and management of high-risk patients with poorer prognosis. More importantly, the association between Patched and Gli1 protein overexpression with poor survival of hepatoblastoma patients was demonstrated for the first time. were significantly correlated with the outcome of patients. Among the three independent parameters, Gli1 protein overexpression, stage of disease, and histological type, the risk of Gli1 protein overexpression was highest, indicating that $\mathrm{Hh}$ signal activation is the most useful prognosis-associating factor in hepatoblastoma. Of course, TNM staging in clinical practice is widely used, and its high prognostic capability is generally accepted. TNM classification is based on the clinicopathologic characteristics, and lack of biological and molecular information, such as marks of proliferation, angiogenesis, invasiveness, apoptosis. Thus, it cannot predict perfectly the outcome for a particular individual. Using Gli-1 as prognostic factors may compensate for the shortage of TNM system, as proven by success of molecular and gene marks in hepatoblastoma for prognosis. Furthermore, analysis can be performed by biopsy even with blood samples from patients. In spite of its variability, it is easily accessible, so it may be helpful for diagnosis and predicting early-stage cancer in patients before radical surgery or in patients who cannot undergo surgery. It was reported that $\mathrm{Hh}$ signaling pathway was related to the cancer cell mobility, migration and invasiveness [21], probably through directly participating in cell migration and angiogenesis processes [22]. The functional properties of such cells might be influenced through cell proliferation, migration, invasion and angiogenesis-promoting effect and apoptosis-inhibiting effect of Gli1 overexpression [23].

In conclusion, molecular evaluation of $\mathrm{Hh}$ signal components have shown that overexpression of Gli1 and Patched correlated with poor clinical outcome in hepatoblastoma. It is tempting to speculate that $\mathrm{Hh}$ pathway may be useful in the evaluation of hepatoblastoma as a decision-making biomarker for predicting treatment efficacy. Prospective studies in a larger population including very early-stage patients should be carried out. Although the functional and clinical significance of the Hh pathway remains to be elucidated, we suggest that Gli-1 expression is a potential diagnostic biomarker and therapeutic target. We also anticipate that the $\mathrm{Hh}$ signal pathway will continue to be a significant target in investigation of cancer therapies.

Acknowledgements: We thank Prof. Kaiyong Tang for providing technical assistance and insightful discussions during the preparation of the manuscript, without whom this work would not have been possible. We thank Dr Xiaoyong
Zhang, at the Wistar Institute, USA, for help with the linguistic revision of the manuscript.

Conflict of Interest Statement: None declared.

Footnotes:

1. The research was supported by key project of Chongqing Natural Science Foundation (CSTC, 2008BA0021), National Natural Science Foundation of China (No. 30973440, 30770950).

2. The authors Xian-Qing Jin and Zhenhua Guo contributed equally to this work.

\section{REFERENCES}

1. Hivama E, Yamaoka H, Matsunaga T, Havashi $Y$, Ando H, Suita S, Horie H, Kaneko M, Sasaki F, Hashizume K, Nakagawara A, Ohnuma N, Yokoyama T. High expression of telomerase is an independent prognostic indicator of poor outcome in hepatoblastoma. Br J Cancer. 2004; 91(5):972-979.

2. Wang LL, Filippi RZ, Zurakowski D, Archibald T, Vargas SO, Voss SD, Shamberger RC, Davies K, Kozakewich H, Perez-Atayde AR. Effects of neoadjuvant chemotherapy on hepatoblastoma: a morphologic and immunohistochemical study. Am J Surg Pathol. 2010;34(3):287-299.

3. Hou JY, Liu HC, Yeh TC, Chen SH, Wang LY, Sheu JC, Liang DC. Long-term treatment results of hepatoblastoma at a single institution in Taiwan. J Pediatr Hematol Oncol. 2009;31(10):718-722.

4. Sakamoto LH, DE Camargo B, Cajaiba M, Soares FA, Vettore AL. MT1G Hypermethylation: A Potential Prognostic Marker for Hepatoblastoma. Pediatr Res. 2010; 67(4):387-393.

5. Oue T, Yoneda A, Uehara S, Yamanaka H, Fukuzawa M. Increased expression of the hedgehog signaling pathway in pediatric solid malignancies. J Pediatr Surg. 2010;45(2): 387-392.

6. von Schweinitz D, Kraus JA, Albrecht S, Koch A, Fuchs J, Pietsch T. Prognostic impact of molecular genetic alterations in hepatoblastoma. Med Pediatr Oncol. 2002;38(2): 104-108.

7. Weber RG, Pietsch T, von Schweinitz D, Lichter P. Characterization of genomic alterations in hepatoblastomas. A role for gains on chromosomes $8 \mathrm{q}$ and 20 as predictors of poor outcome. Am J Pathol. 2000;157(2):571-578.

8. Kameda C, Tanaka H, Yamasaki A, Nakamura M, Koga K, Sato N, Kubo M, Kuroki S, Tanaka M, Katano M. The Hedgehog pathway is a possible therapeutic target for patients with estrogen receptor-negative breast cancer. Anticancer Res. 2009;29(3):871-879.

9. Liao X, Siu MK, Au CW, Wong ES, Chan HY, Ip PP, Ngan HY, Cheung AN. Aberrant activation of hedgehog signaling pathway in ovarian cancers: effect on prognosis, cell invasion and differentiation. Carcinogenesis. 2009; 30(1):131-140.

10. Yoshikawa R, Nakano Y, Tao L, Koishi K, Matsumoto T, Sasako M, Tsujimura T, Hashimoto-Tamaoki T, Fujiwara Y. Hedgehog signal activation in oesophageal cancer patients undergoing neoadjuvant chemoradiotherapy. Br J Cancer. 2008;98(10):1670-1674.

11. Lauth M, Toftgård R. The Hedgehog pathway as a drug target in cancer therapy. Curr Opin Investig Drugs. 2007;8(6):457-461.

12. Haas JE, Muczynski KA, Krailo M, Ablin A, Land V, Vietti TJ, Hammond GD. Histopathology and prognosis in childhood hepatoblastoma and hepatocarcinoma. Cancer. 1989; 64(5):1082-1095.

13. Zhang D, Qiu L, Jin X, Guo Z, Guo C. Nuclear factorkappaB inhibition by parthenolide potentiates the efficacy of Taxol in non-small cell lung cancer in vitro and in vivo. Mol Cancer Res. 2009;7(7):1139-1149. 
14. Yagui-Beltrán A, He B, Raz D, Kim J, Jablons DM. Novel therapies targeting signaling pathways in lung cancer. Thorac Surg Clin. 2006;16(4):379-396.

15. Mori Y, Okumura T, Tsunoda S, Sakai Y, Shimada Y. Gli-1 expression is associated with lymph node metastasis and tumor progression in esophageal squamous cell carcinoma. Oncology. 2006;70(5):378-389.

16. Chen X, Horiuchi A, Kikuchi N, Osada R, Yoshida J, Shiozawa T, Konishi I. Hedgehog signal pathway is activated in ovarian carcinomas, correlating with cell proliferation: it's inhibition leads to growth suppression and apoptosis. Cancer Sci. 2007;98(1):68-76.

17. Ferretti E, Di Marcotullio L, Gessi M, Mattei T, Greco A, Po A, De Smaele E, Giangaspero F, Riccardi R, Di Rocco C, Pazzaglia S, Maroder M, Alimandi M, Screpanti I, Gulino A. Alternative splicing of the ErbB-4 cytoplasmic domain and its regulation by hedgehog signaling identify distinct medulloblastoma subsets. Oncogene. 2006;25(55): 7267-7273.

18. Nolan-Stevaux O, Lau J, Truitt ML, Chu GC, Hebrok M, Fernández-Zapico ME, Hanahan D. GLI1 is regulated through Smoothened-independent mechanisms in neoplastic pancreatic ducts and mediates PDAC cell survival and transformation. Genes Dev. 2009;23(1):24-36.

19. Yoshizaki A, Nakayama T, Naito S, Wen CY, Sekine I. Expressions of sonic hedgehog, patched, smoothened and Gli-1 in human intestinal stromal tumors and their correlation with prognosis. World J Gastroenterol. 2006; 12(35):5687-5691.
20. Kim KH, Kim JM, Choi YL, Shin YK, Lee HC, Seong IO, Kim BK, Chae SW, Chung YS, Kim SH. Expression of sonic hedgehog signaling molecules in normal, hyperplastic and carcinomatous endometrium. Pathol Int. 2009; 59(5):279-287.

21. Hochman E, Castiel A, Jacob-Hirsch J, Amariglio N, Izraeli S. Molecular pathways regulating pro-migratory effects of Hedgehog signaling. J Biol Chem. 2006;281(45): 33860-33870.

22. Davidson B, Goldberg I, Gotlieb WH, Kopolovic J, Risberg B, Ben-Baruch G, Reich R. Coordinated expression of integrin subunits, matrix metalloproteinases (MMP), angiogenic genes and Ets transcription factors in advanced-stage ovarian carcinoma: a possible activation pathway? Cancer Metastasis Rev. 2003;22(1):103-115.

23. Sodek KL, Ringuette MJ, Brown TJ. MT1-MMP is the critical determinant of matrix degradation and invasion by ovarian cancer cells. Br J Cancer. 2007;97(3):358-367.

Received: April 24, 2010 / Accepted: July 19, 2010

Address for correspondence:

Chunbao Guo, M.D., Ph.D.

Department of Hepatobiliary Surgery

Children's Hospital of Chongqing Medical University

22 Zhongshan Rd.

Chongqing, 400014, P.R. China

Tel.: +86-23-63870729;

E-mail: gchunbao@yahoo.com.cn 